\title{
Electromagnetic Dissociation as a Tool for Nuclear Structure and Astrophysics
}

\author{
Gerhard Baur $^{\mathrm{a}, 1}$ Kai Hencken ${ }^{\mathrm{b}, 2}$ Dirk Trautmann ${ }^{\mathrm{b}, 3}$ \\ Stefan Typel $^{\mathrm{c}, 4}$ Hermann H. Wolter ${ }^{\mathrm{c}, 5}$

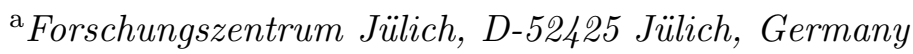 \\ ${ }^{\mathrm{b}}$ Universität Basel, CH-4056 Basel, Switzerland \\ ${ }^{\mathrm{c}}$ Universität München, D-85748 Garching, Germany
}

\begin{abstract}
Coulomb dissociation is an especially simple and important reaction mechanism. Since the perturbation due to the electric field of the nucleus is exactly known, firm conclusions can be drawn from such measurements. Electromagnetic matrix elements and astrophysical S-factors for radiative capture processes can be extracted from experiments. We describe the basic theory, new results concerning higher order effects in the dissociation of neutron halo nuclei, and briefly review the experimental results obtained up to now. Some new applications of Coulomb dissociation for nuclear astrophysics and nuclear structure physics are discussed.
\end{abstract}

Key words: Electromagnetic (Coulomb) dissociation, nuclear structure, nuclear astrophysics, radiative capture

PACS: 24.10.-i, 21.10.-k, 95.30Cq

\section{Introduction}

With increasing beam energy higher lying states of nuclei can be excited with the Coulomb excitation mechanism. This can lead to Coulomb dissociation, in addition to Coulomb excitation of particle bound states, for a review see , e.g.,

1 E-mail: g.baur@fz-juelich.de

2 E-mail: k.hencken@unibas.ch

3 E-mail: dirk.trautmann@unibas.ch

${ }^{4}$ E-mail: stefan.typel@physik.uni-muenchen.de

${ }^{5}$ E-mail: hermann.wolter@physik.uni-muenchen.de 
[1]. Such investigations are also well suited for secondary (radioactive) beams. In this review we start with a general discussion of Coulomb dissociation. Due to the time-dependent electromagnetic field the projectile is excited to a bound or continuum state, which can subsequently decay. We briefly mention the very large effects of electromagnetic excitation in relativistic heavy ion collisions. If $1^{\text {st }}$ order electromagnetic excitation is the dominant effect, experiments can directly be interpreted in terms of electromagnetic matrixelements, which also enter e.g. in radiative capture cross-sections The question of higher order effects is therefore very important. We present new results for a simple and realistic model for Coulomb dissociation of neutron halo nuclei. We show that these effects are reassuringly small. After a short review of results obtained for nuclear structure as well as nuclear astrophysics, we discuss new possibilities, like the experimental study of two-particle capture. We close with conclusions and an outlook.

\section{General Remarks on Electromagnetic Dissociation}

Coulomb excitation is a very useful tool to determine nuclear electromagnetic matrixelements. This is of interest for nuclear structure and nuclear astrophysics [2,3]. Multiple electromagnetic excitation can also be important. We especially mention two aspects: It is a way to excite new nuclear states, like the double phonon giant dipole resonance [3]; but it can also be a correction to the one-photon excitation [4-6].

In the equivalent photon approximation the cross section for an electromagnetic process is written as

$$
\sigma=\int \frac{d \omega}{\omega} n(\omega) \sigma_{\gamma}(\omega)
$$

where $\sigma_{\gamma}(\omega)$ denotes the appropriate cross section for the photo-induced process and $n(\omega)$ is the equivalent photon number. For sufficiently high beam energies it is well approximated by

$$
n(\omega)=\frac{2}{\pi} Z^{2} \alpha \ln \frac{\gamma v}{\omega R}
$$

where $R$ denotes some cut-off radius. More refined expressions, which take into account the dependence on multipolarity, beam velocity or Coulomb-deflection, are available in the literature $[1,5,7]$. The theory of electromagnetic excitation is well developed for nonrelativistic, as well as relativistic projectile velocities. In the latter case an analytical result for all multipolarities was obtained in Ref. [7]. The projectile motion was treated classically in a 
straight-line approximation. On the other hand, in the Glauber theory, the projectile motion can be treated quantally $[1,6]$. This gives rise to characteristic diffraction effects. The main effect is due to the strong absorption at impact parameters less than the sum of the two nuclear radii.

\section{Electromagnetic Excitation in Relativistic Heavy Ion Collisions}

Electromagnetic excitation is also used at relativistic heavy ion accelerators to obtain nuclear structure information. Recent examples are the nuclear fission studies of radioactive nuclei [8] and photofission of ${ }^{208} \mathrm{~Pb}$ [9]. Cross-sections for the excitation of the giant dipole resonance ("Weizsäcker-Williams process") at the forthcoming relativistic heavy ion colliders $\mathrm{RHIC}$ and $\mathrm{LHC}(\mathrm{Pb}-\mathrm{Pb})$ at CERN are huge [10,11], of the order of $100 \mathrm{~b}$ for heavy systems (Au-Au or $\mathrm{Pb}-\mathrm{Pb}$ ). In colliders, the effect is considered to be mainly a nuisance, because the excited particles are lost from the beam. On the other hand, the effect will also be useful as a luminosity monitor by detecting the neutrons in the forward direction. Specifically one will measure the neutrons which will be produced after the decay of the giant dipole resonance which is excited in each of the ions(simultaneous excitation). Since this process has a steeper impact parameter dependence than the single excitation cross-section, there is more sensitivity to the cut-off radius and to nuclear effects. For details and further Refs., see [11].

\section{Higher Order Effects and Postacceleration}

Higher order effects can be taken into account in a coupled channels approach, or by using higher order perturbation theory. The latter involves a sum over all intermediate states $n$ considered to be important. Another approach is to integrate the time-dependent Schrödinger equation directly for a given model Hamiltonian [12-15]. If the collision is sudden, one can neglect the time ordering in the usual perturbation approach. Then the interaction can be summed to infinite order. Intermediate states $n$ do not appear explicitly.

Higher order effects were recently studied in [16], where further references also to related work can be found. Since full Coulomb wave functions in the initial and final channels are used there, the effects of higher order in $\eta_{\text {coul }}=\frac{Z Z_{c} e^{2}}{\hbar v}$ are taken into account to all orders. Expanding this T-matrixelement for the process $Z+a \rightarrow Z+c+n$ in this parameter $\eta_{\text {coul }}$ one obtains the Born 
approximation

$$
T=f_{\text {coul }} \frac{2 D_{0}}{\pi^{2}}\left(\frac{1}{q_{a}^{2}-\left(\vec{q}_{n}+\vec{q}_{c}\right)^{2}}+\frac{m_{c}}{\left(m_{n}+m_{c}\right)\left(q_{c}^{2}-\left(\vec{q}_{n}-\vec{q}_{a}\right)^{2}\right)}\right)
$$

where the zero-range constant $D_{0}$ is given by $\frac{\hbar^{2}}{2 \mu} \sqrt{8 \pi \eta}$. The parameter $\eta$ is related to the binding energy $E_{0}$ by $E_{0}=\frac{\hbar^{2} \eta^{2}}{2 \mu}$. The quantitiy $f_{\text {coul }}$ is related to the elastic Coulomb scattering amplitude up to an irrelevant phase factor. It is given by $f_{\text {coul }}=\frac{2 \eta_{\text {coul }} q_{a}}{q_{\text {coul }}^{2}}$, where the momentum transfer is given by $\vec{q}_{\text {coul }}=$ $\vec{q}_{a}-\vec{q}_{c}-\vec{q}_{n}$. This expression is somehow related to the Bethe-Heitler formula for bremsstrahlung. The Bethe-Heitler formula has two terms, one of which corresponds to a Coulomb interaction between the electron and the target followed by the photon emission, and another one, where the photon is emitted first and then the electron scatters from the nucleus. Here we have a Coulomb scattering of the incoming particle followed by breakup $a=(c+n) \rightarrow c+n$ and another term, where the projectile $a$ breaks up into $c+n$, and subsequently, $c$ is scattered on the target $Z$. In the case of bremsstrahlung it is well known [17] that even for $\eta_{\text {coul }} \gg 1$ one obtains the Born approximation result as long as the scattering is into a narrow cone in the forward direction. This leads one to suspect that higher order effects are not very large in the case of high energy Coulomb dissociation, where the fragments are emitted into the forward direction.

For a small enough Coulomb push $q_{\text {coul }}$ the T-matrixelement, eq. (3) can be expanded in $q_{\text {coul }} / q$ to give in lowest order (using energy conservation)

$$
T=f_{\text {coul }} \frac{2 D_{0}}{\pi^{2}} \frac{\left(m_{n}\right)^{2} m_{c}}{\left(m_{n}+m_{c}\right)^{3}} \frac{2 \vec{q} \cdot \vec{q}_{\text {coul }}}{\left(\eta^{2}+q^{2}\right)^{2}}
$$

The relative momentum between $n$ and $c$ is given by $\vec{q}=\frac{m_{c} \vec{q}_{n}-m_{n} \vec{q}_{c}}{m_{n}+m_{c}}$. This formula is in remarkable agreement with the usual $1^{\text {st }}$ order treatment of electromagnetic excitation in the semiclassical approximation. In this approach the T-matrix is proportional to the elastic scattering amplitude times an excitation probability. This excitation amplitude is e.g. given explicitly for the present zero range model in [4].

We investigate higher order effects in the model of [4-6]. In a zero range model for the neutron-core interaction, analytical results were obtained for $1^{\text {st }}$ and $2^{\text {nd }}$ order electromagnetic excitation for small values of the adiabaticity parameter $\xi$. We are especially interested in collisions with small impact parameters where higher order effects tend to be larger than for the very distant ones. In this case, the adiabaticity parameter $\xi$ is small. For $\xi=0$ (sudden approximation) we have a closed form solution, where higher order effects are taken into account to all orders. In eq. (37) of [4] the angle integrated breakup probability 
is given. We expand this expression in the strength parameter $y=\frac{2 Z Z_{c} e^{2} m_{n}}{\hbar v\left(m_{n}+m_{c}\right) b \eta}$, where $b$ is the impact parameter. We define $x=\frac{q}{\eta}$ where the wave number $\mathrm{q}$ is related to the energy $E_{\mathrm{rel}}$ of the continuum final state by $E_{\mathrm{rel}}=\frac{\hbar^{2} q^{2}}{2 \mu}$. In leading order (LO) we obtain

$$
\frac{d P_{L O}}{d q}=\frac{16}{3 \pi \eta} y^{2} \frac{x^{4}}{\left(1+x^{2}\right)^{4}}
$$

The next to leading order (NLO) expression is proportional to $y^{4}$ and contains a contribution from the $2^{\text {nd }}$ order E1 amplitude and a contribution from the interference of $1^{\text {st }}$ and $3^{\text {rd }}$ order. We find

$$
\frac{d P_{N L O}}{d q}=\frac{16}{3 \pi \eta} y^{4} \frac{x^{2}\left(5-55 x^{2}+28 x^{4}\right)}{15\left(1+x^{2}\right)^{6}}
$$

The integration over $\mathrm{x}$ and the impact parameter $\mathrm{b}$ can also be performed analytically in good approximation. For details see [18]. We can easily insert the values for the Coulomb dissociation experiments on ${ }^{11} \mathrm{Be}$ and ${ }^{19} \mathrm{C}[19,20]$ in the present formulae. We find that the ratio of the NLO contribution to the LO contribution in the case of Coulomb dissociation on ${ }^{19} \mathrm{C}[20]$ is given by $-10 \%$. This is to be compared to the results of [16] where a value of about $-35 \%$ was found. The reason for these differences has to be investigated further.

Postacceleration is a higher order effect. A semiclassical model might suggest that the parallel momentum distribution of the core is shifted towards larger values due to an "extra Coulomb push", see e.g. [21]. However, this turns out to be wrong. In the sudden approximation, the core-neutron binding is negligible. Also on its way towards the target the core alone( and not the bound neutron- core system) feels the Coulomb interaction. Thus there is no extra Coulomb push. Corrections due to finite values of $\xi$ were studied in [4]. They were found to be a rather delicate quantal interference effect depending only on the phase shift of the neutron s-wave. The effects are quite small and it is worth mentioning that no postacceleration effects were found in a recent ${ }^{11}$ Be Coulomb dissociation experiment [22].

\section{Discussion of some experimental results for nuclear structure and astrophysics}

Coulomb dissociation of exotic nuclei is a valuable tool to determine electromagnetic matrix-elements between the ground state and the nuclear continuum. The excitation energy spectrum of the ${ }^{10} \mathrm{Be}+\mathrm{n}$ system in the Coulomb dissociation of the one-neutron halo nucleus ${ }^{11} \mathrm{Be}$ on a $\mathrm{Pb}$ target at $72 \cdot \mathrm{A} \mathrm{MeV}$ 
was measured [19]. Low lying E1-strength was found. The Coulomb dissociation of the extremely neutron-rich nucleus ${ }^{19} \mathrm{C}$ was recently studied in a similar way [20]. The neutron separation energy of ${ }^{19} \mathrm{C}$ could also be determined to be $530 \pm 130 \mathrm{keV}$. Quite similarly, the Coulomb dissociation of the 2 n-halo nucleus ${ }^{11} \mathrm{Li}$ was studied in various laboratories [23-25]. In an experiment at MSU [26], the correlations of the outgoing neutrons were studied. Within the limits of experimental accuracy, no correlations were found.

In nuclear astrophysics, radiative capture reactions of the type $b+c \rightarrow a+\gamma$ play a very important role. They can also be studied in the time-reversed reaction $\gamma+a \rightarrow b+c$, at least in those cases where the nucleus $a$ is in the ground state. As a photon beam, we use the equivalent photon spectrum which is provided in the fast peripheral collision. Reviews, both from an experimental as well as theoretical point of view have been given [2], so we want to concentrate here on a few points.
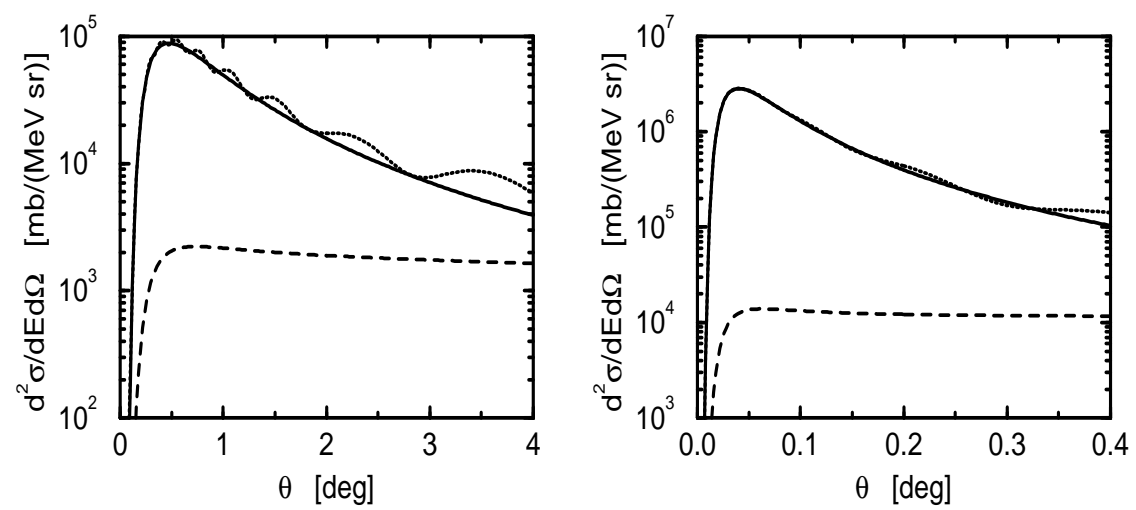

Fig. 1. Coulomb dissociation cross section of ${ }^{8} \mathrm{~B}$ scattered on ${ }^{208} \mathrm{~Pb}$ as a function of the scattering angle for projectile energies of $46.5 A \cdot \mathrm{MeV}$ (left) and $250 \mathrm{~A} \cdot \mathrm{MeV}$ (right) and a ${ }^{7}$ Be-p relative energy of $0.3 \mathrm{MeV}$. First order results E1 (solid line), E2 (dashed line) and E1+E2 excitation including nuclear diffraction (dotted line). [From Figs. 4 and 5 of Ref. [6].]

The ${ }^{6} \mathrm{Li}$ Coulomb dissociation into $\alpha+\mathrm{d}$ has been a test case of the method, see Ref. [2]. This is of importance since the $d(\alpha, \gamma)^{6} \mathrm{Li}$ radiative capture is the only process by which ${ }^{6} \mathrm{Li}$ is produced in standard primordial nucleosynthesis models. There has been new interest in ${ }^{6} \mathrm{Li}$ as a cosmological probe in recent years, mainly because the sensitivity for searches for ${ }^{6} \mathrm{Li}$ has been improving. It has been found in metal-poor halo stars at a level exceeding even optimistic estimates of how much ${ }^{6} \mathrm{Li}$ could have been made in standard big bang nucleosynthesis. For more discussion on this see [27].

The ${ }^{7} \mathrm{Be}(\mathrm{p}, \gamma){ }^{8} \mathrm{~B}$ radiative capture reaction is relevant for the solar neutrino problem. It determines the production of ${ }^{8} \mathrm{~B}$ which leads to the emission of high energy neutrinos. There are direct reaction measurements, for a recent one see Refs. [28]. Coulomb dissociation of ${ }^{8} \mathrm{~B}$ has been studied at RIKEN [29], MSU [30] and GSI [31]. Theoretical calculations are shown in Fig. 1. It is seen 
that E1 excitation is large and peaked at very forward angles. E2 excitation is also present, with a characteristically different angular distribution. Nuclear diffraction effects are small. Altogether it is quite remarkable that completely different experimental methods with possibly different systematic errors lead to results that are quite consistent.

\section{Possible New Applications of Coulomb dissociation for nuclear astrophysics}

Nucleosynthesis beyond the iron peak proceeds mainly by the r- and s-processes (rapid and slow neutron capture) [32,33]. To establish the quantitative details of these processes, accurate energy-averaged neutron-capture cross sections are needed. Such data provide information on the mechanism of the neutroncapture process and time scales, as well as temperatures involved in the process. The data should also shed light on neutron sources, required neutron fluxes and possible sites of the processes (see Ref. [32]). The dependence of direct neutron capture on nuclear structure models was investigated in Ref. [34]. The investigated models yield capture cross-sections sometimes differing by orders of magnitude. This may also lead to differences in the predicted astrophysical r-process paths. Because of low level densities, the compound nucleus model will not be applicable.

With the new radioactive beam facilities (either fragment separator or ISOLtype facilities) some of the nuclei far off the valley of stability, which are relevant for the r-process, can be produced. In order to assess the r-process path, it is important to know the nuclear properties like $\beta$-decay half-lifes and neutron binding energies. Sometimes, the waiting point approximation $[32,33]$ is introduced, which assumes an $(\mathrm{n}, \gamma)$ - and $(\gamma, \mathrm{n})$-equilibrium in an isotopic chain. It is generally believed that the waiting point approximation should be replaced by dynamic r-process flow calculations, taking into account $(\mathrm{n}, \gamma),(\gamma, \mathrm{n})$ and $\beta$-decay rates as well as time-varying temperature and neutron density. In slow freeze-out scenarios, the knowledge of $(n, \gamma)$ cross sections is important.

In such a situation, the Coulomb dissociation can be a very useful tool to obtain information on $(n, \gamma)$-reaction cross sections on unstable nuclei, where direct measurements cannot be done. Of course, one cannot and need not study the capture cross section on all the nuclei involved; rather there will be some key reactions of nuclei close to magic numbers. It was proposed [35] to use the Coulomb dissociation method to obtain information about $(n, \gamma)$ reaction cross sections, using nuclei like ${ }^{124} \mathrm{Mo},{ }^{126} \mathrm{Ru},{ }^{128} \mathrm{Pd}$ and ${ }^{130} \mathrm{Cd}$ as projectiles. The optimum choice of beam energy will depend on the actual neutron binding energy. Since the flux of equivalent photons has essentially an $\frac{1}{\omega}$ de- 
pendence, low neutron thresholds are favourable for the Coulomb dissociation method. Note that only information about the $(\mathrm{n}, \gamma)$ capture reaction to the ground state is possible with the Coulomb dissociation method. The situation is reminiscent of the loosely bound neutron-rich light nuclei, like ${ }^{11} \mathrm{Be},{ }^{11} \mathrm{Li}$ and ${ }^{19} \mathrm{C}$.

A new field of application of the Coulomb dissociation method can be two nucleon capture reactions. Evidently, they cannot be studied in a direct way in the laboratory. Sometimes this is not necessary, when the relevant information about resonances involved can be obtained by other means (transfer reactions, etc.), like in the triple $\alpha$-process.

Two-neutron capture reactions in supernovae neutrino bubbles are studied in Ref. [37]. In the case of a high neutron abundance, a sequence of two-neutron capture reactions, ${ }^{4} \mathrm{He}(2 \mathrm{n}, \gamma){ }^{6} \mathrm{He}(2 \mathrm{n}, \gamma){ }^{8} \mathrm{He}$ can bridge the $A=5$ and 8 gaps. The ${ }^{6} \mathrm{He}$ and ${ }^{8} \mathrm{He}$ nuclei may be formed preferentially by two-step resonant processes through their broad $2^{+}$first excited states [37]. Dedicated Coulomb dissociation experiments can be useful, see [38]. Another key reaction can be the ${ }^{4} \mathrm{He}(\alpha \mathrm{n}, \gamma)$ reaction [37]. The ${ }^{9} \mathrm{Be}(\gamma, \mathrm{n})$ reaction has been studied directly (see Ref. [39]) and the low energy $s_{\frac{1}{2}}$ resonance is clearly established.

In the rp-process, two-proton capture reactions can bridge the waiting points [40-42]. From the ${ }^{15} \mathrm{O}(2 \mathrm{p}, \gamma){ }^{17} \mathrm{Ne},{ }^{18} \mathrm{Ne}(2 \mathrm{p}, \gamma){ }^{20} \mathrm{Mg}$ and ${ }^{38} \mathrm{Ca}(2 \mathrm{p}, \gamma){ }^{40} \mathrm{Ti}$ reactions considered in Ref. [41], the latter can act as an efficient reaction link at conditions typical for X-ray bursts on neutron stars. A ${ }^{40} \mathrm{Ti} \rightarrow \mathrm{p}+\mathrm{p}+$ ${ }^{38} \mathrm{Ca}$ Coulomb dissociation experiment should be feasible. The decay with two protons is expected to be sequential rather than correlated ("2 $\mathrm{He} "$-emission). The relevant resonances are listed in Table XII of Ref. [41]. In Ref. [42] it is found that in X-ray bursts $2 \mathrm{p}$-capture reactions accelerate the reaction flow into the $Z \geq 36$ region considerably. In Table 1 of Ref. [42] nuclei, on which $2 \mathrm{p}$-capture reactions may occur, are listed; the final nuclei are ${ }^{68} \mathrm{Se},{ }^{72} \mathrm{Kr},{ }^{76} \mathrm{Sr}$, ${ }^{80} \mathrm{Zr},{ }^{84} \mathrm{Mo},{ }^{88} \mathrm{Ru},{ }^{92} \mathrm{Pd}$ and ${ }^{96} \mathrm{Cd}$ (see also Fig. 8 of Ref. [40]). It is proposed to study the Coulomb dissociation of these nuclei in order to obtain more direct insight into the 2 p-capture process.

\section{Conclusions}

Peripheral collisions of medium and high energy nuclei (stable or radioactive) passing each other at distances beyond nuclear contact and thus dominated by electromagnetic interactions are important tools of nuclear physics research. The intense source of quasi-real (or equivalent) photons has opened a wide horizon of related problems and new experimental possibilities especially for the present and forthcoming radioactive beam facilities to investigate 
efficiently photo-interactions with nuclei (single- and multiphoton excitations and electromagnetic dissociation).

\section{Acknowledgments}

We have enjoyed collaboration and discussions on the present topics with very many people. We are especially grateful to C. A. Bertulani, H. Rebel, F. Rösel, and R. Shyam.

\section{References}

[1] C. A. Bertulani and G. Baur, Phys. Rep. 163 (1988) 299

[2] G. Baur, C. A. Bertulani and H. Rebel, Nucl. Phys. A458 (1986) 188; G. Baur and H. Rebel, J. Phys. G: Nucl. Part. Phys. 20 (1994) 1; Ann. Rev. Nucl. Part. Sci. 46 (1996) 321

[3] G. Baur and C. A. Bertulani, Phys. Lett. B174 (1986) 23

[4] S. Typel and G. Baur, Nucl. Phys. A573 (1994) 486

[5] S. Typel and G. Baur, Phys. Rev. C50 (1994) 2104

[6] S. Typel, H. H. Wolter and G. Baur, Nucl. Phys. A613 (1997) 147

[7] A. Winther and K. Alder, Nucl. Phys. A319 (1979) 518

[8] K.-H. Schmidt et al., Nucl. Phys. A665 (2000) 221, T. Enqvist et al., Nucl.Phys. A658(1999) 47

[9] M. C. Abreu et al., Phys. Rev. C59 (1999) 876

[10] G. Baur and C. A. Bertulani, Nucl. Phys. A505 (1989) 835

[11] G. Baur, K. Hencken and D. Trautmann, J. Phys. G: Nucl. Part. Phys. 24 (1998) 1657

[12] V. S. Melezhik and D. Baye, Phys. Rev. C59 (1999) 3232

[13] H. Esbensen, G. F. Bertsch and C. A. Bertulani, Nucl. Phys. A581 (1995) 107

[14] H. Utsunomia, Y. Tokimoto, T. Yamagata, M. Ohta, Y. Aoki, K. Hirota, K. Ieki, Y. Iwata, K. Katori, S. Hamada, Y.-W. Lui, R. P. Schmitt, S. Typel and G. Baur, Nucl. Phys. A654 (1999) 928c

[15] S. Typel, H. H. Wolter, Z. Naturforsch. 54 a (1999) 63 
[16] J. A. Tostevin, Paper presented at: 2nd International Conference on Fission and Neutron Rich Nuclei, St. Andrews, Scotland, June 28 - July 2 1999, ed. J. H. Hamilton et al., World Scientific, Singapore 2000

[17] L. D. Landau and E. M. Lifshitz, Quantenelektrodynamik, Lehrbuch der Theoretischen Physik Band 4 (Berlin: Akademie 1986)

[18] S. Typel and G. Baur, Higher Order Effects in Electromagnetic Dissociation of Neutron Halo Nuclei, in preparation

[19] T. Nakamura et al., Phys. Lett. B331 (1994) 296

[20] T. Nakamura et al., Phys. Rev. Lett. 83 (1999) 1112

[21] G. Baur, C.A. Bertulani and D.M. Kalassa Nucl.Phys.A550(1992)527

[22] J.E. Bush et al. Phys. Rev. Lett. 81(1998) 61

[23] T. Kobayashi et al., Phys. Lett. B232 (1989) 51

[24] S. Shimoura et al., Phys. Lett. B348 (1995) 29

[25] M. Zinser et al., Nucl. Phys. A619 (1997) 151

[26] K. Ieki, A. Galonski et al., Phys. Rev. C54 (1996) 1589

[27] K. M. Nollett, M. Lemoine, and D. N. Schramm, Phys. Rev. C56 (1997) 1144; K. M. Nollett et al., nucl-th/0006064

[28] F. Hammache et al., Phys. Rev. Lett. 80 (1998) 928

[29] T. Motobayashi et al., Phys. Rev. Lett. 73 (1994) 2680

[30] J. H. Kelley et al., Phys. Rev. Lett. 77 (1996) 5020

[31] N. Iwasa et al., Phys. Rev. Lett. 83 (1999) 2910

[32] C. E. Rolfs and W. S. Rodney, Cauldrons in the Cosmos, The University of Chicago Press (1988)

[33] J. J. Cowan, F.-K. Thielemann and J. W. Truran, Phys. Rep. 208 (1991) 267

[34] T. Rauscher et al., Phys. Rev. C57 (1998) 2031

[35] M. Gai, ISOL workshop, Columbus/Ohio, July 30 - August 1, 1997

[36] J. von Schwarzenberg et al., Phys. Rev. C53 (1996) R2598

[37] J. Görres, H. Herndl, I. J. Thompson and M. Wiescher, Phys. Rev. C52 (1995) 2231

[38] T. Aumann et al., Phys. Rev. C59 (1999) 1252

[39] F. Ajzenberg-Selove, Nucl. Phys. A490 (1988) 1

[40] NuPECC Report, Nuclear and Particle Astrophysics, July 16, 1997, I. Baraffe et al., F.-K. Thielemann (convener)

[41] J. Görres, M. Wiescher and F.-K. Thielemann, Phys. Rev. C51 (1995) 392

[42] H. Schatz et al., Phys. Rep. 294 (1998) 167 